\title{
The association of childhood socio-economic position and psychological distress in adulthood: is it mediated by adult socio-economic position?
}

\author{
Sarah K Mckenzie ${ }^{1,2}$ \\ Kristie Carter ${ }^{1}$ \\ Tony Blakely ${ }^{1}$ \\ Sunny Collings ${ }^{2}$ \\ ${ }^{1}$ Health Inequalities Research Programme, Department of Public Health, University of Otago, Wellington, New Zealand \\ ${ }^{2}$ Social Psychiatry \& Population Mental Health Research Unit, University of Otago, Wellington, New Zealand \\ sarah.mckenzie@otago.ac.nz
}

(Received February 2010 Revised August 2010)

\section{Abstract}

There is substantial evidence that lower socio-economic position (SEP) is associated with poorer mental health outcomes. However, uncertainties exist about the origins of socioeconomic gradients in mental health problems and the relative contributions of both childhood and adult SEP. In this study we assess the association of childhood SEP with psychological distress in adulthood and investigate how much of this association is mediated by adult SEP. Data for this cross-sectional analysis came from Wave 3 of the Survey of Family, Income and Employment (SoFIE) in New Zealand ( $n=14,470)$. Childhood SEP was measured using parental occupation recalled at age 10. Non-specific psychological distress was assessed using the Kessler 10 scale (K10). Adult SEP was measured using five socio-economic indicators (area deprivation, household income, wealth, labour market activity, education). The association of childhood SEP with psychological distress before and after controlling for confounders and adult SEP indicators was determined using logistic regression, with the $K 10$ dichotomised at low/moderate versus high/very high. Sensitivity analyses included birth cohort and sex. There was a weak inverse relationship between increasing proportion of psychological distress with lower childhood SEP. Adjusted for age, sex and ethnicity, respondents with low compared to high childhood SEP had 1.35 greater odds of reporting high psychological distress (95\% Cl 1.13-1.60). Adjustment for adult mediating SEP variables led to a $77 \%$ reduction in the excess odds ratio to 1.08 (95\% Cl 0.90 1.29). The relationship did not significantly differ by birth cohort or sex. This finding is consistent with the current evidence that socio-economic circumstances in adulthood are important determinants of inequalities in adult mental health and mediate much of the association of childhood SEP with adult psychological distress.

Keywords: psychological distress, mental health, Kessler 10, childhood socio-economic position, adulthood socio-economic position 


\section{Introduction}

The literature on the existence of adult socioeconomic gradients in mental health is extensive (Dohrenwend, Levav et al 1992; Weich and Lewis 1998; Miech, Caspi et al 1999; Fryers, Melzer et al 2003; Lorant, Deliege et al 2003; Stansfeld, Head et al 2003; Muntaner, Eaton et al 2004; Skapinakis, Weich et al 2006; Butterworth, Rodgers et al 2009). Socioeconomic disparities in adult mental health have been shown in a number of populations (Dohrenwend, Levav et al 1992; Weich and Lewis 1998; Weich and Lewis 1998; Miech, Caspi et al 1999; Fryers, Melzer et al 2003; Skapinakis, Weich et al 2006; Carter, Blakely et al 2009) and across a range of mental disorders, including schizophrenia, anti-social personality disorder and affective disorders (Power, Stansfeld et al 2002). However, for the more common mental disorders and general psychological distress, which make up the majority of the burden of mental illness in the community, the findings are inconsistent. Most studies have, however, shown significantly higher rates of specific disorders, such as depression, anxiety or a combination of both among lower socio-economic groups (Stansfeld, Head et al 1998; Miech, Caspi et al 1999; Lorant, Deliege et al 2003; Stansfeld, Head et al 2003; Lorant, Croux et al 2007). Those groups with less education, more unemployment and lower income or material assets, tend to have higher prevalence rates of the common mental disorders (Fryers, Melzer et al 2003).

Despite this, the origins of the socio-economic differences observed in inequalities in adult mental health remain under investigated, particularly the association and relative contributions of earlier and later SEP conditions. A key question from the life course perspective is whether the higher rates of adult ill health, observed in the lower socio-economic groups, reflect influences that took place earlier in the life course, in adolescence or earlier stages of adulthood, or whether they reflect more recent influences. The identification of socio-economic pathways that link early life experiences to social inequalities in adult mental health is therefore an important line of enquiry within the life course framework.

Previous investigations of the effects of childhood and adult SEP on health have tended to focus exclusively on physical health outcomes such as coronary heart disease (Singh-Manoux, Ferrie et al 2004), self-rated health (Laaksonen, Rahkonen et al 2005), biological risk factors such as blood pressure, body mass index and cholesterol (Melchior, Moffitt et al 2007; Power, Atherton et al 2007), cause-specific mortality (Davey Smith, Hart et al 1998; Galobardes, Lynch et al 2008) and all-cause mortality (Kuh, Hardy et al 2002). These studies comprise a growing body of literature showing that whilst poor socio-economic circumstances during childhood are associated with adverse health outcomes in adulthood, adjusting for measures of current adult SEP can explain some or all of the association (i.e. much if not all of the association of childhood socio-economic position with adult health is mediated by adult socio-economic position). Similar studies, exploring the contributions of both early and later life SEP to adult mental health, are not as common and their findings inconsistent. Some have demonstrated a significant association between adverse childhood SEP and poorer mental health independently of adult SEP, concluding that socio-economic differences, certainly for depression, originate early in life and do not appear to be fully mediated by adult socio-economic position (Gilman, Kawachi et al 2002; Luo and Waite 2005; Power, Atherton et al 2007). However, other studies have shown that poor mental health is more strongly associated with adult than childhood SEP (Harper, Lynch et al 2002; Poulton, Caspi et al 2002). It is unclear whether these effects are due to direct effects from childhood SEP on adult mental health or whether they are due to an indirect effect through adult SEP.

We might expect a direct effect of childhood SEP on adult mental health as hypothesised by the 'critical periods model'. According to this model, an exposure acting during a specific period of development (such as childhood SEP) may have lasting or lifelong effects on the structure or function of organs, tissues and body systems that are not modified in any dramatic way by later life experience (Kuh and Ben-Shlomo 2004). Also known as 'biological programming', this is the basis of the 'fetal origins of adult disease' hypotheses (Barker 1998). The socio-economic environment in childhood could affect exposure during gestation, infancy, and childhood to adverse causal factors which are part of long-term biological 
processes. These factors are generally associated with various aspects of childhood development and wellbeing such as social competence, emotion processing; cognitive development; psychosocial behaviours; and the development of biological stressresponses (Bradley and Corwyn 2002; Repetti, Taylor et al 2002). Children from low socio-economic families are more likely to begin life in poor health, experience more biological and psychosocial risk factors; more stress; more fetal and birth complications and have elevated rates of emotional and behavioural problems than those born into higher socio-economic groups (Power and Matthews 1997; Poulton, Caspi et al 2002; Graham and Power 2004).

We might also expect an indirect effect of childhood SEP on adult psychological distress as hypothesised by the 'chain of risk' or the 'pathways model' (Kuh and Ben-Shlomo 2004). According to these models a sequence of linked exposures leads to impaired function and increased risk of ill health because one bad experience or exposure leads to another and so on. Each exposure in the chain of risk may not only increase the risk of subsequent exposure, but may also have an independent 'additive effect 'on later function or disease (Kuh and Ben-Shlomo 2004). It is also argued that there is continuity of family socio-economic circumstances or social class (including grandparent social class) during childhood and adolescence through to adulthood. The socio-economic circumstances of the family are responsible for influencing children's access to social and economic resources, and relate strongly to the child's opportunities for education and learning experiences (Kuh, Power et al 2004). There is also substantial evidence from cohort studies that education is an important determinant of subsequent occupational career, and thus opportunities for ensuring income and favourable living conditions in adulthood (Kuh and Wadsworth 1991; Kuh, Head et al 1997; Graham 2007). Adult socio-economic circumstances have in turn been shown to affect mental health status through factors such as unemployment, deprivation, or poor social networks and support (Power and Manor 1992; Lorant, Deliege et al 2003). The influence of the early socio-economic environment on adult socio-economic trajectories therefore is a hypothesised major indirect pathway through which childhood SEP may exert an effect on adult health (Kuh, Power et al 2004; Singh-Manoux, Ferrie et al 2004). Adult SEP is argued to act as a mediating variable, as not only is it heavily influenced by childhood SEP, but is itself also predictive of later health outcomes.

Our study uses data from a survey in New Zealand (NZ) to address the following research questions (i) Is there a direct association between a retrospective measure of childhood SEP and psychological distress in adulthood? and if so (ii) How much of the association is mediated by adult SEP through an indirect pathway? (childhood SEP $\rightarrow$ adult SEP $\rightarrow$ psychological distress). We also examine whether this association differs by sex or birth cohort.

\section{Methods}

This study is a cross-sectional analysis utilising data from the Survey of Families, Income and Employment (SoFIE) conducted by Statistics New Zealand (Wave 1 to 4 version 6) (Carter, Cronin et al 2009). SoFIE is New Zealand's first national survey designed to study income, family type and employment and how they change over a period of 8 years. It is a nationally representative fixed-panel longitudinal survey of the usually resident population living in private dwellings in New Zealand in 2002. SoFIE used the standard Statistics New Zealand sampling frame used for other household surveys (Carter, Cronin et al 2009). In SoFIE, face to face interviews are used to collect information annually on income levels, sources and changes; and on major influences on income such as employment and education experiences, household and family status and changes, demographic factors and health status. Information on assets and liabilities is collected every two years (Waves 2, 4, 6 and 8). At Waves 3, 5 and 7 an array of health questions, collecting information of health-related quality of life, psychological distress, co-morbidities (e.g. stroke, diabetes, and injury), lifestyle factors, perceived stress and primary care usage are asked. In this analysis, data were restricted to adults ( 15 years or older) who answered questions at Wave 3 , as this wave also included questions about parental occupation. 
The quality of longitudinal data through time is heavily affected by attrition as it occurs at each wave of the survey, resulting in fewer and fewer people who have complete records over the course of the survey. The initial SoFIE sample comprised approximately 11,500 randomly-selected responding private households (response rate of $77 \%$ ) with data collected from 22,165 individuals aged 15 and over at Wave 1. All individuals who were asked and responded to the Wave 1 interview were original sample members (OSMs). Children under the age of 15 at Wave 1 were interviewed as OSMs from Wave 2 onwards once they had turned 15. All household members over the age of 15 were eligible to take part. By Wave 2 the sample had reduced to just over 20,000 responding OSMs (89\%) and by Wave 3 (2004), 18,955 responding adults answered the SoFIE Health questionnaire ( $82 \%$ of Wave 1 adult OSMs) (Statistics New Zealand 2008). The highest attrition rates between wave 1 and 3 were observed in respondents living in the most deprived areas (26.6\%), with no qualifications (20.3\%), in the lowest income group (29\%), lowest quintile for wealth (17.6\%), unemployed (31.4\%) and in the minority ethnic groups (Asian 38.4\%, Maori 30.9\% and Pacific 29.4\%).

Of the 18,955 adults who answered the health questionnaire at Wave 3 , parental occupation was missing for 4,090 respondents. When the sample was further restricted by excluding those adults with missing information on psychological distress ( $\mathrm{n}=$ $125)$ the final sample size reduced to 14,740 . Respondents in the final sample were more likely to be aged over 35 years, identify as New Zealand/European (70.4\%), report higher educational qualifications, income and wealth and be less likely to report living in a deprived area compared to the original Wave 1 sample.

\section{Measures}

\section{Childhood socio-economic position}

As part of the health module (Wave 3) of SoFIE, respondents were asked to recall the occupation of both parents when they were aged 10. These occupations were coded using the 1999 version of the New Zealand Standard Classification of Occupations
(NZSCO99) which provides a standardised framework for classifying occupational data (Statistics New Zealand 2001). Our measure of childhood SEP was derived by mapping the highest occupational code of both parents to the New Zealand Socio-economic Index of Occupational Status (NZSEI 1996) (Davis, McLeod et al 1997) thereby creating a measure of childhood socio-economic status for each respondent. The NZSEI is a census-derived, occupation based measure of SES based on a 'return to human capital' model of social stratification (Davis, McLeod et al 1997; Davis, McLeod et al 1999; Davis, Jenkin et al 2003; Davis, Jenkin et al 2004). NZSEI is a linear scale of ranked occupation, produced using an algorithm involving age, income and education. Thus, variations in occupational orders translate into variations in social stratification and differentiation in lifestyles and life chances (Davis, McLeod et al 1997; Davis, McLeod et al 1999). The socio-economic scores are scaled from 10 (representing the occupational group at the lowest) to 90 (the occupational group at the highest ends of the socio-economic hierarchy). For our analysis, NZSEI scores were split into three discrete socio-economic groups using cutpoints based on the $33^{\text {rd }}$ and $66^{\text {th }}$ percentile splits of the distribution of scores in our sample, creating similar score ranges to previous work by Davis et al. The distribution of childhood SEP groups over NZSEI-96 were as follows: low (10-30), medium (31-46) and high (47-90) childhood SEP groups. Sensitivity analyses for our final modelling strategy conducted using the NZSEI-scores continuously, rather than in tertiles, produced similar results.

\section{Psychological distress}

Mental health was assessed at Wave 3 using the 10-item Kessler psychological distress Scale (K10). This scale has been developed specifically for assessing the prevalence of general psychological distress symptoms at the community level, and is increasingly being used in population mental health research. It has been validated in multiple settings (Andrews and Slade 2001) and has sound psychometric properties (Kessler, Andrews et al 2002). In SoFIE, respondents were asked how often they had experienced each of the 10 states elicited by the K10 (mainly anxiety and depressive symptoms) in the four weeks prior to interview. The frequency with 
which each of the 10 items was experienced was recorded using a five-point Likert scale ranging from 1 (none of the time) to 5 (all the time). This score was then summed with increasing scores reflecting an increasing degree of psychological distress. As there is no agreed standard for determining cut-off points for levels of psychological distress, and various interpretations of scoring have been used to date, the scores in our study were grouped according to the criteria developed by Andrews and Slade (2001) into four levels of psychological distress: low (10-15); moderate (16-21); high (22-29); very high distress $(\geq 30)$. For the current analysis, the K10 was dichotomised at low to moderate levels of distress (K10 $\leq 21)$ versus high to very high levels of psychological distress (K10 $\geq 22)$ (Phongsavan, Chey et al 2006). High to very high levels of psychological distress have been shown to be associated with clinical diagnoses of anxiety and affective disorders (Andrews and Slade 2001; Oakley Browne, Wells et al 2010). Using alternative K10 cutpoints, or multichotomous logistic regression, did not substantially alter our findings and how much of the relationship was mediated by adult SEP.

\section{Adult socio-economic position}

We used five measures of the participant's current SEP at the time of the Wave 3 interview to reflect different aspects of both material and social resources in adulthood (Galobardes, Shaw et al 2006). For our research questions, it is important that we adjust as completely as possible for adult socioeconomic position; hence including multiple measures of adult socio-economic position is desirable. These include area deprivation, education, household income and labour market activity. The NZ deprivation (NZDep2001) index provides a small area deprivation score composed of census variables which reflect aspects of material and social deprivation (Salmond and Crampton 2002). NZDep2001 information was divided into quintiles where NZDepQ1 is the least deprived and NZDepQ5 is the most deprived. Education, which captures earlyto mid-life adult socio-economic position, was measured using the respondent's maximum educational qualification over the three waves and categorised as nil, high school, post-school vocational (diploma/certificate) or, bachelor degree or higher qualification(s). Equivalised household income (CPI adjusted to 2002), a measure of material resources at the household level, was divided into quintiles using the mean household income across the first three waves. Labour market activity was used as a measure of workforce status, as these conditions are associated with material resources, for example those who are unemployed may have a lack of resources. Labour market activity was defined as employed, not employed but seeking work, or not employed and not seeking work at the interview date. Wealth is a measure of total assets and can include financial and physical assets such as housing, investments or pensions. Net worth (taken from Wave 2 of SoFIE) was calculated by subtracting the total value of all liabilities from the total value of all assets for individuals and couples, and categorised into quintiles (Carter, Hayward et al 2008).

\section{Covariates}

Respondents' sex and age was asked at the initial interview and then checked with the respondents at subsequent waves. At each wave, every adult was also asked their self-identified ethnicity. Participants were asked to 'choose as many responses as you need to say which ethnic groups you belong to', from a list of fifteen possible groups. For analyses we used prioritised ethnicity where each respondent was allocated to a single ethnic group using the priority recording system (Allan 2001). Ethnicity was defined as New Zealand/European (those primarily of European descent), Māori (the indigenous people of New Zealand), Pacific (those of Pacific Island descent e.g. Samoan, Cook Island, Fijian), Asian (those of Southeast Asia, China or Indian descent) and other (non NZ/European, non-Maori, non-Pacific and nonAsian).

\section{Statistical methods}

Associations between childhood SEP and adult psychological distress were investigated using multiple logistic regression models to calculate odds ratios (OR) and $95 \%$ confidence intervals $(\mathrm{Cl})$. Firstly, the odds of experiencing high to very high psychological distress (score of 22+) in the low and medium childhood SEP groups, as compared with the high childhood SEP group, were calculated with 
adjustment for the confounders sex, age and ethnicity (model 1). Secondly, respondent's own NZDep2001 score, education, income, labour market activity and wealth were added to the confounder adjusted model, one at a time (model 2). Finally the model was fully adjusted for confounders and all five adult SEP indicators simultaneously (model 3 ). The fully adjusted ORs (from model 3) were compared with those from model 1 by calculating the percentage change in the excess OR (ORModel 2-ORModel 1)/ (ORModel 1-1) in the low compared to high childhood SEP group. As a test of whether the change in strength of the association of low childhood SEP with psychological distress was statistically significant, we conducted a Hausman test (Hausman 1978; Greenland 2008). This involved calculating the difference in the beta coefficients and constructing 95\% confidence intervals before (model 1 ) and after adjusting for mediating variables (model 3 ).

\section{Sensitivity analyses}

It is argued that the association between childhood SEP and psychological distress could, potentially, vary by sex, as the determinants of mental health differ between males and females (Piccinelli and Wilkinson 2000; Artazcoz, Benach et al 2004). It could also vary by birth cohort, which, given the cross-sectional nature of our data, would be equivalent to variation by age in this study (Kuh, BenShlomo et al 2003). This could possibly be due to increasing measurement error of parental occupation with older subjects, or perhaps more substantively, due to true cohort variation in effects. Thus, to account for these possible influences, or effect modification, three birth cohorts were created covering three time periods (1920-1950,1950$1970,1970-1990)$ using the respondent's age at Wave 3. Models 1 (age, sex, ethnicity adjusted) and 3 (fully adjusted) were re-run, stratified by sex and birth cohort, and the Wald Statistic test was applied to test for heterogeneity between the sex and age strata.

All data were analysed on unit-level data in the Statistics New Zealand data laboratory using SAS 8.2. All tabular numbers of respondents presented in this paper are random rounded to the nearest multiple of five as per Statistics New Zealand confidentiality protocol. Analyses were run both with and without longitudinal weights (taking into account attrition and weighting to the New Zealand population at 2002); there was no difference in the results so we present the unweighted results.

\section{Results}

The demographic and socio-economic characteristics of the study sample in relation to categories of psychological distress (K10) are presented in Table 1 . Overall, $79 \%$ of the study sample reported low levels of psychological distress (K10 score between 10 and 15). This distribution is heavily skewed but is the same as found in the recent NZ Health Survey 2006/07 and the NZ Mental Health Survey (Oakley Browne, Wells et al 2006; Ministry of Health 2008). There was a slightly higher proportion of high to very high psychological distress present in the low childhood SEP group as compared to the high childhood SEP group. Females made up a little more than half of the sample (54.1\%) and reported higher levels of psychological distress. The majority of the sample identified as NZ/European ethnicity, with Pacific respondents reporting higher levels of psychological distress than any other ethnic group. All adult SEP indicators illustrated a linear relationship with psychological distress, i.e. more respondents in poorer income and wealth quintiles, or living in more deprived areas, reported high to very high levels of psychological distress. 
Table 1. Characteristics of SoFIE Health respondents by psychological distress (K10) at Wave 3 ( $n=14,740)$

Category of psychological distress (total K10 score) (row \%)

\begin{tabular}{|c|c|c|c|c|c|}
\hline & & Low 10-15 & Moderate 16- & High 22-29 & Very high $\geq 3$ \\
\hline Childhood SEP & $\mathrm{n}$ & (\%) & $21(\%)$ & (\%) & (\%) \\
\hline Low & 6,520 & 78.5 & 14.7 & 5.1 & 1.6 \\
\hline Medium & 3,610 & 79.2 & 14.3 & 5.0 & 1.5 \\
\hline High & 4,610 & 80.7 & 14.1 & 4.1 & 1.1 \\
\hline \multicolumn{6}{|l|}{ Sex } \\
\hline Female & 7,975 & 77.5 & 15.4 & 5.2 & 1.9 \\
\hline Male & 6,765 & 81.5 & 13.2 & 4.2 & 1.0 \\
\hline \multicolumn{6}{|l|}{ Ethnicity } \\
\hline NZ/European & 11,980 & 80.7 & 13.8 & 4.2 & 1.3 \\
\hline Maori & 1,340 & 74.3 & 16.8 & 7.1 & 2.2 \\
\hline Asian & 725 & 75.9 & 17.2 & 4.8 & 2.1 \\
\hline Pacific & 450 & 70.0 & 18.9 & 10.0 & 2.2 \\
\hline Other & 245 & 71.4 & 16.3 & 8.2 & 4.1 \\
\hline \multicolumn{6}{|l|}{ Age } \\
\hline $15-24$ & 2,035 & 74.0 & 18.7 & 6.1 & 1.5 \\
\hline $25-34$ & 2,015 & 76.9 & 16.9 & 4.5 & 1.7 \\
\hline $35-44$ & 3,030 & 78.5 & 14.4 & 5.3 & 1.7 \\
\hline $45-54$ & 2,925 & 82.1 & 12.5 & 4.3 & 1.4 \\
\hline $55-64$ & 2,230 & 83.2 & 11.4 & 4.0 & 1.8 \\
\hline $65+$ & 2,495 & 80.4 & 14.2 & 4.6 & 0.8 \\
\hline \multicolumn{6}{|l|}{ Highest educational qualification } \\
\hline Degree or higher & 2,235 & 83.9 & 12.8 & 2.7 & 0.7 \\
\hline Post school vocational & 5,170 & 80.5 & 14.0 & 4.1 & 1.5 \\
\hline School qualification & 3,920 & 78.2 & 15.7 & 5.1 & 1.1 \\
\hline No qualification & 3,415 & 76.1 & 14.6 & 6.9 & 2.3 \\
\hline \multicolumn{6}{|l|}{ NZDeprivation } \\
\hline NZDepQ1(least) & 3,215 & 86.3 & 10.6 & 2.3 & 0.6 \\
\hline NZDepQ2 & 3,150 & 81.9 & 12.7 & 4.3 & 1.0 \\
\hline NZDepQ3 & 2,745 & 79.6 & 14.4 & 4.9 & 1.6 \\
\hline NZDepQ4 & 3,100 & 75.2 & 17.4 & 5.3 & 1.8 \\
\hline NZDepQ5(most) & 2,520 & 72.2 & 17.5 & 7.7 & 2.6 \\
\hline \multicolumn{6}{|l|}{ Household income } \\
\hline q1: low $\quad-<\$ 21,080$ & 1,485 & 71.4 & 17.5 & 8.4 & 3.0 \\
\hline$q 2: \$ 21,080-<\$ 34,010$ & 3,265 & 74.0 & 17.8 & 6.0 & 2.5 \\
\hline q3: $\$ 34,010-<\$ 49,380$ & 2,825 & 77.2 & 15.6 & 6.0 & 1.4 \\
\hline$q 4: \$ 49,380-<\$ 72,280$ & 3,290 & 83.1 & 12.5 & 3.5 & 0.8 \\
\hline q5: $\$ 72,280-<$ high & 3,865 & 85.6 & 11.3 & 2.7 & 0.5 \\
\hline \multicolumn{6}{|l|}{ Labour market activity } \\
\hline Not employed, looking for work & 235 & 63.8 & 19.1 & 12.8 & 4.3 \\
\hline Not employed, not looking for work & 4,595 & 73.6 & 16.9 & 7.1 & 2.2 \\
\hline Working & 9,910 & 82.4 & 13.1 & 3.5 & 1.1 \\
\hline \multicolumn{6}{|l|}{ Wealth } \\
\hline Q1: low $-<\$ 25,590$ & 3,860 & 72.7 & 17.9 & 7.0 & 2.3 \\
\hline Q2: $\$ 25,590-<\$ 70,315$ & 2,515 & 76.1 & 16.1 & 5.8 & 2.2 \\
\hline Q3: $\$ 70,315-<\$ 128,090$ & 2,545 & 81.1 & 13.4 & 4.3 & 1.2 \\
\hline Q4: \$128,090 - > \$232,935 & 2,635 & 83.5 & 12.5 & 3.2 & 0.6 \\
\hline Q5: \$232,935 - high & 2,675 & 87.7 & 9.9 & 2.2 & 0.4 \\
\hline
\end{tabular}


Table 2. Description of respondent's socio-economic position in childhood and adulthood

\begin{tabular}{|c|c|c|c|c|c|}
\hline & & Childh & socioeconom & osition & \\
\hline Adult socioeconomic indicators & $\mathbf{n}$ & Low (\%) & Medium (\%) & High (\%) & $\begin{array}{c}\text { Pearson } \\
\text { correlation } \\
\text { coefficients }\end{array}$ \\
\hline Highest educational qualification & & & & & \\
\hline Degree or higher & 2,230 & 27.1 & 20.4 & 52.5 & \\
\hline Post school vocational qualification & 5,165 & 42.8 & 27.0 & 30.2 & \\
\hline School qualification & 3,915 & 41.5 & 24.1 & 34.4 & \\
\hline No qualification & 3,420 & 60.7 & 23.8 & 15.5 & $0.19 *$ \\
\hline NZ Deprivation & & & & & \\
\hline NZDepQ1(least) & 3,215 & 37.6 & 23.6 & 38.7 & \\
\hline NZDepQ2 & 3,145 & 42.3 & 24.0 & 33.7 & \\
\hline NZDepQ3 & 2,750 & 43.3 & 25.5 & 31.3 & \\
\hline NZDepQ4 & 3,095 & 46.8 & 24.7 & 28.4 & \\
\hline NZDepQ5(most) & 2,520 & 52.8 & 25.2 & 22.0 & $0.12 *$ \\
\hline Household income & & & & & \\
\hline q1: low $-<\$ 21,080$ & 1,485 & 48.8 & 24.9 & 26.3 & \\
\hline$q 2: \$ 21,080-<\$ 34,010$ & 3,275 & 51.8 & 24.6 & 23.7 & \\
\hline q3: $\$ 34,010-<\$ 49,380$ & 2,825 & 46.0 & 25.3 & 28.7 & \\
\hline$q 4: \$ 49,380-<\$ 72,280$ & 3,290 & 42.6 & 25.1 & 32.4 & \\
\hline q5: $\$ 72,280-<$ high & 3,870 & 36.2 & 23.3 & 40.6 & $0.10^{*}$ \\
\hline Labour market activity & & & & & \\
\hline Not employed, looking for work & 235 & 42.6 & 27.7 & 29.8 & \\
\hline Not employed, not looking for work & 4,595 & 47.9 & 24.6 & 27.5 & \\
\hline Working & 9,905 & 42.6 & 24.4 & 33.0 & $0.06 *$ \\
\hline Wealth & & & & & \\
\hline Q1: low $-<\$ 25,590$ & 3,865 & 40.4 & 23.7 & 36.0 & \\
\hline Q2: \$25,590 - <\$70,315 & 2,520 & 45.6 & 25.0 & 29.4 & \\
\hline Q3: $\$ 70,315-<\$ 128,090$ & 2,540 & 47.2 & 25.8 & 27.0 & \\
\hline Q4: $\$ 128,090-<232,935$ & 2,625 & 46.1 & 26.7 & 27.2 & \\
\hline Q5: \$232,935 - high & 2,680 & 44.4 & 22.4 & 33.2 & 0.01 \\
\hline
\end{tabular}


Table 2 shows the distribution of childhood and adult SEP measures for the study sample. Respondents reporting low childhood SEP were more likely to reside in the most deprived areas (NZDepQ5), have no educational qualifications, report lower household income and lower wealth and be not employed/ not looking for work, in comparison to those reporting higher childhood SEP. Childhood SEP was most strongly graded with educational qualifications. $27.1 \%$ of those from a low childhood SEP reported a degree or higher, whereas $60.7 \%$ reported no qualification. This was in contrast to the weaker grading observed for NZDep, household income, wealth and labour market activity. A potential problem is collinearity between the measures of SEP across the life course. Pearson correlation coefficients between childhood SEP and all five adult SEP indicators in our sample were low, and ranged from 0.01 to 0.19 with all, except wealth, being statistically significantly correlated $(p<0.0001)$.

Table 3 presents results from logistic regression analyses. In model 1 (adjusted for confounders) both low and medium childhood SEP groups were significantly associated with high levels of psychological distress. The relative odds of reporting high psychological distress among those with low compared to high childhood SEP was $1.35(95 \% \mathrm{Cl}$ 1.13-1.60). The odds comparing moderate to high childhood SEP were similar to low SEP. The association between childhood SEP and psychological distress persisted after separately adjusting for each adult SEP indicators individually except education (Model 2). All measures except labour market activity attenuated the excess odds ratios. Both education and income were the strongest mediators of the association, reducing the OR independently by $63 \%$ and $40 \%$ respectively. Adjustment for all five adult SEP indicators simultaneously in addition to confounders, (Model 3) led to a 77\% reduction in the excess OR from 1.35 to 1.08 ( $95 \% \mathrm{Cl} 0.90$ to 1.29). The Hausman test showed the reduction in the beta coefficients before and after adjusting for adult SEP measures was statistically significant indicating that the change in strength of the association of low childhood SEP with psychological distress after adjustment was statistically significant.

The results of our sensitivity analyses investigating whether there is effect modification by birth cohort and sex are shown in Table 4. There was no statistically significant difference between the relationship of childhood SEP on psychological distress by birth cohort or by sex. 
Table 3. Odds ratios $(\mathrm{OR})$ and confidence intervals $(\mathrm{Cl})$ for logistic regression modelling of childhood SEP on adult psychological distress*

\begin{tabular}{|c|c|c|c|c|c|c|c|c|c|c|c|c|c|c|}
\hline \multirow[b]{3}{*}{ Childhood SEP } & \multirow{2}{*}{\multicolumn{2}{|c|}{$\begin{array}{l}\text { Model } 1 \text { adjusted } \\
\text { for respondents } \\
\text { age, sex and } \\
\text { ethnicity } \\
\text { OR }(95 \% \mathrm{Cl})\end{array}$}} & \multicolumn{2}{|c|}{ education } & \multicolumn{2}{|c|}{ NZDeprivation } & \multicolumn{2}{|r|}{ income } & \multicolumn{2}{|c|}{$\begin{array}{l}\text { labour market } \\
\text { activity }\end{array}$} & \multicolumn{2}{|r|}{ wealth } & \multicolumn{2}{|c|}{$\begin{array}{l}\text { age, sex, ethnicity } \\
\text { and all measures of } \\
\text { adult SEP }\end{array}$} \\
\hline & & & \multicolumn{2}{|c|}{ OR $(95 \% \mathrm{Cl})$} & \multicolumn{2}{|c|}{ OR (95\% Cl) } & \multicolumn{2}{|c|}{ OR (95\% Cl) } & \multicolumn{2}{|c|}{ OR $(95 \% \mathrm{Cl})$} & \multicolumn{2}{|c|}{ OR (95\% Cl) } & \multicolumn{2}{|c|}{ OR (95\% Cl) } \\
\hline & & & & & & & & & & & & & & \\
\hline Low & 1.35 & $(1.13-1.60)$ & 1.13 & $(0.95-1.35)$ & 1.25 & $(1.05-1.49)$ & 1.21 & $(1.02-1.44)$ & 1.35 & $(1.13-1.60)$ & 1.27 & $(1.07-1.51)$ & 1.08 & $(0.90-1.29)$ \\
\hline Medium & 1.36 & $(1.12-1.65)$ & 1.21 & $(1.00-1.47)$ & 1.28 & $(1.06-1.56)$ & 1.26 & $(1.04-1.53)$ & 1.36 & $(1.12-1.65)$ & 1.31 & $(1.08-1.59)$ & 1.15 & $(0.95-1.41)$ \\
\hline High (ref) & 1.00 & & 1.00 & & 1.00 & & 1.00 & & 1.00 & & 1.00 & & 1.00 & \\
\hline \multicolumn{15}{|l|}{ Age } \\
\hline 15-24 (ref) & 1.00 & & 1.00 & & 1.00 & & 1.00 & & 1.00 & & 1.00 & & 1.00 & \\
\hline $25-34$ & 0.78 & $(0.60-1.02)$ & 0.87 & $(0.67-1.13)$ & 0.75 & $(0.58-0.98)$ & 0.78 & $(0.60-1.02)$ & 0.93 & $(0.72-1.22)$ & 1.00 & $(0.77-1.31)$ & 1.03 & $(0.78-1.37)$ \\
\hline $35-44$ & 0.91 & $(0.72-1.15)$ & 0.96 & $(0.76-1.21)$ & 0.93 & $(0.74-1.17)$ & 0.93 & $(0.74-1.17)$ & 1.16 & $(0.91-1.47)$ & 1.56 & $(1.21-2.02)$ & 1.52 & (1.16-1.99) \\
\hline $45-54$ & 0.73 & $(0.48-0.94)$ & 0.74 & $(0.57-0.95)$ & 0.74 & $(0.58-0.95)$ & 0.82 & $(0.65-1.05)$ & 0.96 & $(0.75-1.23)$ & 1.55 & $(1.18-2.04)$ & 1.42 & $(1.07-1.90)$ \\
\hline $55-64$ & 0.74 & $(0.58-0.96)$ & 0.66 & $(0.51-0.87)$ & 0.74 & $(0.57-0.96)$ & 0.76 & $(0.58-0.99)$ & 0.79 & $(0.61-1.03)$ & 1.72 & $(1.28-2.32)$ & 1.21 & $(0.88-1.65)$ \\
\hline $65+$ & 0.75 & $(0.58-0.97$ & 0.62 & $(0.48-0.82)$ & 0.71 & $(0.55-0.92)$ & 0.60 & $(0.46-0.79)$ & 0.46 & $(0.35-0.61)$ & 1.66 & $(1.24-2.22)$ & 0.70 & $(0.50-0.98)$ \\
\hline \multicolumn{15}{|l|}{ Sex } \\
\hline Male (ref) & 1.00 & & 1.00 & & 1.00 & & 1.00 & & 1.00 & & 1.00 & & 1.00 & \\
\hline Female & 1.40 & $(1.21-1.61)$ & 1.39 & $(1.20-1.60)$ & 1.39 & $(1.20-1.60)$ & 1.32 & $(1.14-1.52)$ & 1.21 & $(1.04-1.39)$ & 1.41 & $(1.23-1.63)$ & 1.24 & $(1.07-1.44)$ \\
\hline \multicolumn{15}{|l|}{ Ethnicity } \\
\hline NZ/European (ref) & 1.00 & & 1.00 & & 1.00 & & 1.00 & & 1.00 & & 1.00 & & 1.00 & \\
\hline Maori & 1.66 & $(1.35-2.06)$ & 1.49 & $(1.20-1.85)$ & 1.31 & $(1.06-1.64)$ & 1.45 & $(1.17-1.79)$ & 1.47 & $(1.18-1.82)$ & 1.29 & $(1.04-1.60)$ & 1.03 & $(0.82-1.30)$ \\
\hline Pacific & 1.93 & $(1.39-2.68)$ & 1.80 & $(1.30-2.51)$ & 1.44 & $(1.03-2.02)$ & 1.60 & $(1.15-2.23)$ & 1.67 & $(1.19-2.32)$ & 1.36 & $(0.97-1.89)$ & 1.10 & $(0.78-1.55)$ \\
\hline Asian & 1.33 & $(0.98-1.81)$ & 1.52 & $(1.12-2.07)$ & 1.20 & $(0.88-1.64)$ & 1.07 & $(0.78-1.46)$ & 1.10 & $(0.81-1.51)$ & 1.15 & $(0.84-1.57)$ & 1.02 & $(0.74-1.40)$ \\
\hline Other & 2.62 & $(1.75-3.90)$ & 2.94 & $(1.96-4.41)$ & 2.55 & $(1.7-3.81)$ & 2.33 & $(1.55-3.49)$ & 2.34 & $(1.56-3.52)$ & 2.14 & $(1.42-3.21)$ & 2.09 & $(1.37-3.18)$ \\
\hline \multicolumn{15}{|c|}{ Highest education qualification } \\
\hline Degree or Higher (ref) & & & 1.00 & & & & & & & & & & 1.00 & \\
\hline Post school vocational & & & 1.89 & $(1.44-2.48)$ & & & & & & & & & 1.51 & $(1.14-2.00)$ \\
\hline School qualification & & & 1.83 & $(1.38-2.43)$ & & & & & & & & & 1.39 & $(1.04-1.86)$ \\
\hline No qualification & & & 3.36 & $(2.53-4.47)$ & & & & & & & & & 2.00 & $(1.48-2.68)$ \\
\hline
\end{tabular}




\section{(Table 3 cont'd)}

\section{NZ Deprivation}

NZDepQ1(least) (ref)

NZDepQ2

NZDepQ3

NZDepQ4

NZDepQ5(most)

Household income

q1: low $-<\$ 21,080$

q2: $\$ 21,080-<34,010$

q3: $\$ 34,010-<\$ 49,380$

q4: $\$ 49,380-<\$ 72,280$

q5: $\$ 72,280-<$ high (ref)

\section{Labour market activity}

\section{Working (ref)}

Not employed, not looking for work

Not employed, looking for work

\section{Wealth}

Q1: low $->\$ 25,590$

Q2: $\$ 25,590-<\$ 70,315$

Q3: $\$ 70,315-<128,090$

Q4: $\$ 128,090-<\$ 232,935$

Q5: $\$ 232,935$ - high (ref)

$\begin{array}{ll}1.00 & \\ 1.76 & (1.36-2.28) \\ 1.99 & (1.53-2.58) \\ 2.31 & (1.80-2.96) \\ 3.04 & (2.36-3.93)\end{array}$

$(1.36-2.28)$
$(1.53-2.58)$
$(1.80-2.96)$
$(2.36-3.93)$

3.45

$2.81 \quad(2.22-3.56)$

$2.33 \quad(1.84-2.96)$

$1.36 \quad(1.06-1.76)$

1.00

1.00

$2.84 \quad(2.41-3.35)$

$3.10 \quad(2.09-4.59)$
1.00

$1.57 \quad(1.20-2.04)$

$1.54 \quad(1.18-2.01)$

$1.59 \quad(1.22-2.06)$

$1.70 \quad(1.30-2.23)$

$1.70 \quad(1.30-2.24)$

$1.49 \quad(1.15-1.92)$

$1.50 \quad(1.20-1.97)$

$1.08 \quad(0.83-1.40)$

1.00

1.00

$2.11 \quad(1.77-2.51)$

$2.16 \quad(1.44-3.23)$

$\begin{array}{llll}4.90 \quad(3.67-6.57) & 2.74 \quad(2.01-3.74)\end{array}$

$3.43 \quad(2.57-4.57) \quad 2.26 \quad(1.67-3.05)$

$2.15 \quad(1.60-2.90) \quad 1.56 \quad(1.14-2.11)$

$1.49 \quad(1.09-2.03) \quad 1.21 \quad(0.88-1.67)$

1.00

* Due to missing values on some covariates, all logistic regression was on $n=14,220$ respondents 
Table 4. Sensitivity analyses of potential effect modifiers sex and birth cohort

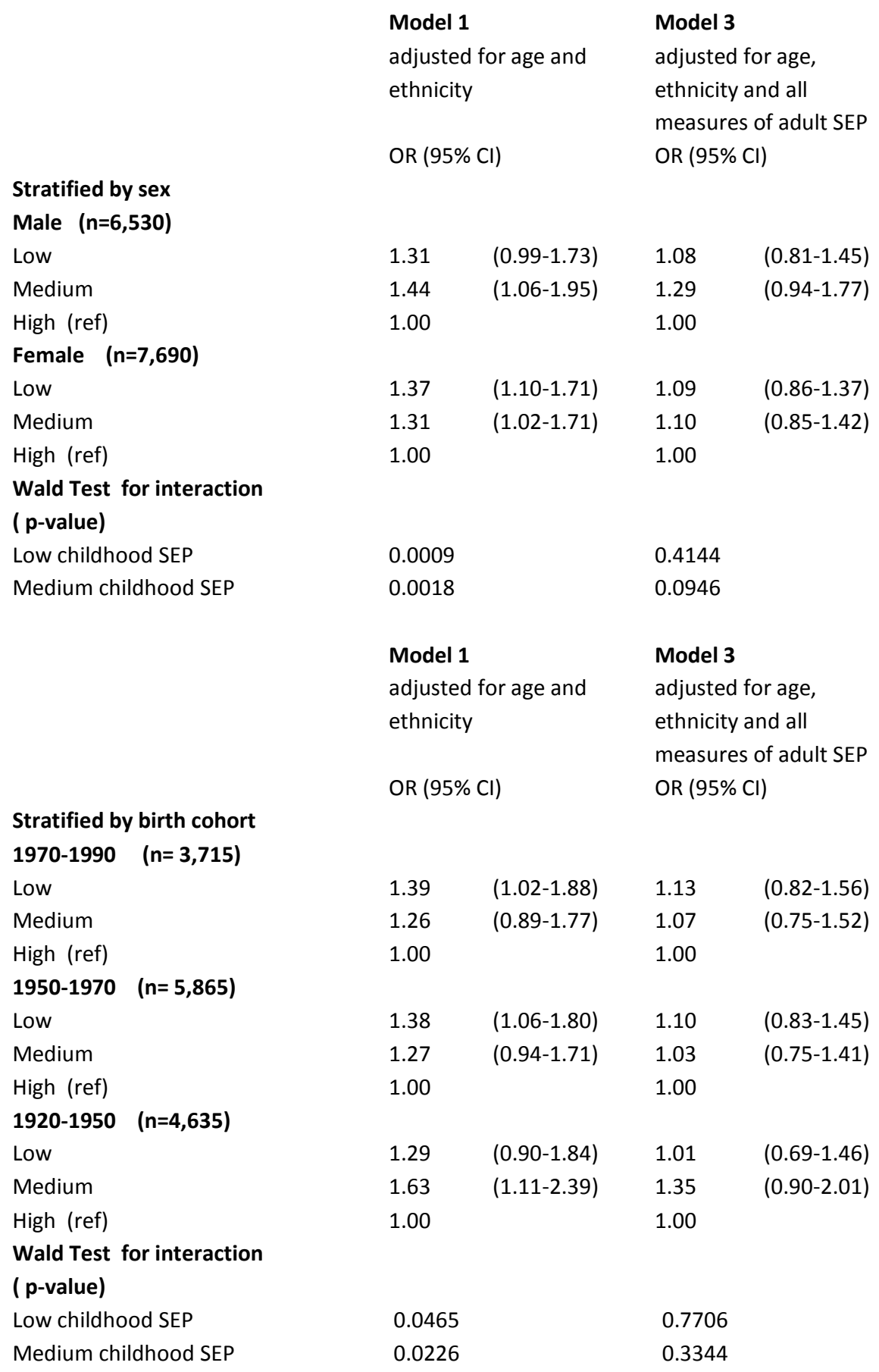




\section{Discussion}

Our study shows that individuals from the lowest childhood socio-economic backgrounds have 35\% greater odds of reporting high to very high psychological distress compared to those individuals from the highest childhood socio-economic backgrounds. Furthermore, more than two-thirds (77\%) of the association of childhood SEP with current psychological distress in adulthood is explained by adult SEP. This is noteworthy as it is a larger proportion mediated (explained by) adult SEP than found in other studies exploring life course SEP and mental health (Gilman, Kawachi et al 2002; Harper, Lynch et al 2002; Luo and Waite 2005; Power, Atherton et al 2007). The association between childhood SEP and psychological distress was largely explained by educational attainment, accounting for $63 \%$ of the association of low childhood SEP on high to very high levels of psychological distress. The proportion mediated by education was greater than for the other adult measures of SEP, suggesting that educational achievement which is influenced by childhood socio-economic position, is the key gateway to socio-economic trajectories that link early life SEP and adult mental health. This is not to say that other socio-economic factors are unimportant. For example, income entered alone explains $40 \%$ of the association. But if education is entered first, the additional contribution of income is small, due to the pathways from childhood socio-economic position to income (presumably), largely going via education. This importance of education is plausible, given it is heavily influenced by parental characteristics such as parental education, income, social class and other household characteristics (Kuh, Power et al 2004).

Additionally, sensitivity analyses showed no interaction of sex or birth cohort in the association and using alternative K10 cutpoints, or multichotomous logistic regression, did not substantially alter our findings and how much of the relationship was mediated by adult SEP.

\section{Strengths and weaknesses}

This study used data from a large nationally representative population in NZ, with the availability of a wide range of measures of adult SEP and a retrospective measure of childhood SEP. Despite these strengths, potential limitations include information bias, unmeasured confounding, selection bias or the possibility of chance findings.

Firstly, the small association observed to start with (OR of 1.35) and the possibility of statistical imprecision in the association of childhood SEP with psychological distress warrants caution. Using the upper and lower confidence limits of Model 1's OR for low childhood SEP (95\% Cl 1.13-1.60) but assuming a constant 0.17 reduction in the OR due to adjustment for adult SEP, then the proportion mediated by adult SEP might range from $28 \%(0.17 / 0.60)$ to at least $100 \%(0.17 / 0.13)$.

Secondly, there is potential for measurement error. It is important to consider measurement errors in the exposure (childhood SEP), mediators (adult SEP) and outcome (psychological distress).

We consider first the impact of measurement error on the exposure-outcome association, without introducing mediators. The use of parental occupation at a single age has been argued to be a weak proxy for more complete information on socioeconomic position spanning the entire childhood period (Glymour 2007). Thus, we have mis-measured our exposure. (Due to the design of the SoFIE study it was not possible to use prospective repeated measures of childhood SEP over time.) Nevertheless, this methodological shortcoming is common to many studies, and does not preclude useful causal inference (Mckenzie and Carter 2009). Additionally, the coding of parental occupation and subsequent assignment to the NZSEI-96 (which meant enforcing socio-economic status levels ascertained in the 1990s on older respondents' parental occupations, which date back to the 1930s) would have introduced further measurement error. This may have led to misclassification of childhood SEP that is greater for older respondents compared to younger respondents. However, as our analysis used a trichotomous measure of childhood SEP, and found no difference by birth cohort, any historical changes in "status" of occupations would need to be substantial enough to cross the two thresholds used to categorise childhood SEP. The critical issue in the mis-measurement of parental SEP is whether it is non-differential and independent of the outcome psychological distress (and the mediator adult SEP). We argue that recall of 
parental occupation, when the adult respondent was aged 10, is a fairly 'concrete' and 'objective' measure. We assume, therefore, that any recall error is not too substantial and neither differential by, nor dependent on, measurement errors on other variables. The subsequent coding and classification to a class may introduce more error, but this too is probably nondifferential and independent of other covariates. Assuming our arguments are correct, we have therefore underestimated the total association of childhood SEP with psychological distress, i.e. the OR of 1.35 is an underestimate.

Consider now measurement error of our mediator, adult SEP, ascertained at the time of interview, using multiple comprehensive measures. We argue that adult SEP is a complex multifaceted construct, and that multiple measures (as we have) are preferable to few in fully adjusting for this construct, and hence this mediation pathway. Put another way, we have more fully adjusted for adult SEP than previous research that has been confined to a single adult SEP indicator such as occupation or education (Lynch, Kaplan et al 1997; Gilman, Kawachi et al 2002; Poulton, Caspi et al 2002; Power, Stansfeld et al 2002; Gilman, Kawachi et al 2003; SinghManoux, Ferrie et al 2004; Melchior, Moffitt et al 2007; Power, Atherton et al 2007). This may explain why we found a greater proportion of the effect of childhood SEP on mental health was mediated by adult SEP, than did other studies, i.e. we simply measured adult SEP more fully than did other studies.
Consider now the joint effect of measurement error of exposure and the mediator. If the target parameter of interest is the proportion of the relationship mediated by adult SEP, and measurement error of both the exposure and mediator are independent and non-differential with respect to the outcome, the proportion of mediation is unchanged with measurement error of the childhood exposure (although the actual ORs are all reduced). However if the mediator is mis-measured, the proportion due to mediation is underestimated (workings available from authors on request). For example, if better measurement of childhood SEP occurred, we might have observed a total OR of 1.70, but (assuming non-differential and non-dependent measurement errors of adult SEP with respect to both childhood SEP and psychological distress) the OR adjusted for adult SEP would have reduced by $77 \%$ to 1.26. Thus, to answer the research question in this paper, it is more important to have an accurate assessment of the adult SEP variables than childhood SEP (assuming non-differential and independence of measurement error).

Thirdly, it is important to note the difficulty with data such as in this study to reliably quantify direct and indirect effects due to unmeasured confounding (Blakely 2002; Cole and Hernan 2002; Hernan and Cole 2009). This is depicted in the following directed acyclic graph (Figure 1).

Figure 1. Directed acyclic graph of the association between childhood SEP, adult SEP and psychological distress

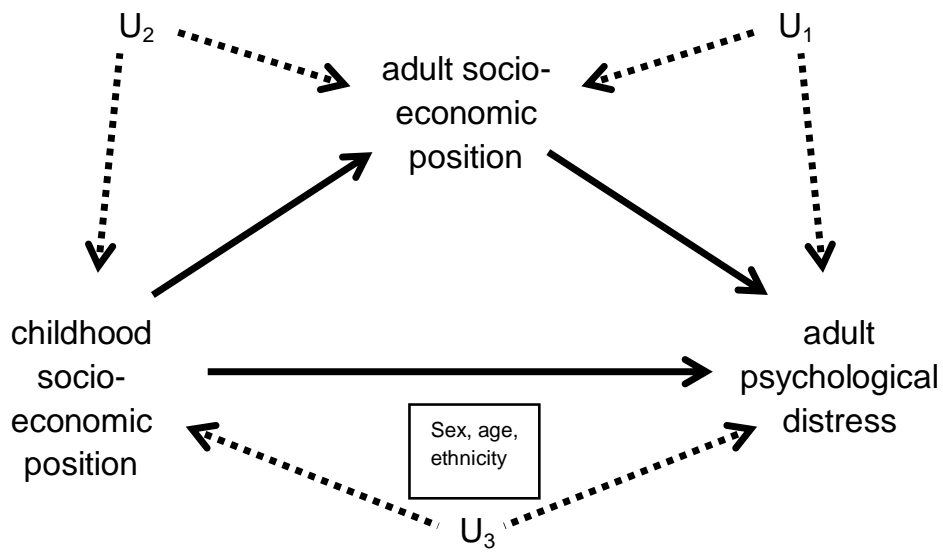


When we adjust for our mediator, adult SEP, it may be that there is unadjusted confounding of the adult SEP-distress association (labelled ' $U_{1}$ ')(Glymour and Greenland 2008). Furthermore, there are also a number of life course measures which can be classed as confounders of the association between childhood SEP and adult mental health status $\left(U_{3}\right)$ and of the association of childhood SEP with adult SEP $\left(U_{2}\right)$, such as childhood health, family history of mental illness and single-parent status. The absence of this information in our study may lead to some bias, probably in the direction to initial overestimation of the total association of childhood SEP with psychological distress; and perhaps some overadjustment for adult SEP when determining indirect effects (if there is strong collider-bias on adult SEP from both $U_{1}$ and $U_{2}$ ). If this were the case, it might be that adult SEP mediates even more (proportionately) of the association - but it is beyond the scope of this paper to empirically model this possibility. We believe in most cases that the magnitude of these confounding biases will not be too severe. For example, it has been shown elsewhere that there needs to be very strong associations of posited additional variables (i.e. $U_{2}$ and $U_{3}$ in the diagram) with both the mediator and outcome of interest, before substantial bias will occur (Greenland 2003; Rothman, Greenland et al 2008).

A limitation of our graph and analysis is that it does not take into account the possible reverse causation of adult psychological distress on adult SEP, where those with pre-existing mental illness drift down the social scale i.e. health selection. In this study we cannot discount the possibility that the association of adult SEP with K10 is overestimated due to health selection, and subsequently, that the proportionate contribution of adult SEP to the childhood SEP and K10 association is also slightly overestimated. We would posit however that some of the adult SEP measures are less subject to selection effects than others. Most formal education, for example, is completed by young adulthood and is predictive of subsequent occupational career. Arguably therefore it is more robust to selection effects (in particular "downward drift") and thereby strengthens our conclusion regarding the mediating effects of education.
The final limitation relates to the selection and attrition of the sample used in our study. Our analysis was restricted to respondents with no missing data on the exposure and outcome at Wave 3 (49\% of the original SoFIE study sample) leading to a sample which was older and potentially more homogenous than the original Wave 1 study population. There is a risk of not being able to obtain information about childhood SEP from a substantial fraction of the very people who are most at risk of poor health. Selection bias could arise if those adults from the lower childhood SEP group who are lost to follow-up, or have missing exposure data, have a different total indirect and direct set of associations between childhood, adult SEP and distress (within strata of covariates). For example, if those adults from the lower childhood SEP group who are lost to follow up or have missing exposure data are also those whom are at the highest risk of psychological distress, then the study will have underestimated the effect of low childhood SEP on the risk of psychological distress. However, for selection bias to arise requires the association of childhood SEP, adult SEP and K10 to differ among those included in the final analyses, compared to those excluded. We do not have any direct evidence whether this is the case or not, as it was not possible to directly estimate the extent and the pattern of 'missingness' on the associations of childhood SEP, adult SEP and K10, as we did not have information on those individuals who were lost to follow-up. Moreover, for the results of our analysis to be pushed towards the null, we would need to see the opposite associations of childhood SEP, adult SEP and psychological distress in the other $50 \%$ of the original study sample who were missing; this seems implausible.

\section{Conclusion}

This research addresses an important life course issue regarding the pathways between childhood socio-economic position and mental health in adulthood. Our results suggest that disadvantaged social environments during childhood may have particularly adverse consequences for adult mental health status, because of their effects on educational achievement and subsequent socio-economic status. A possible explanation for our finding is that the 
direct effects of early socio-economic environment via pathways other than adult SEP are less important for psychological distress than that of the later adult SEP pathway, and hence after adjustment for adult SEP the effect of childhood SEP is no longer significant. This does not refute the early origins argument per se. Rather this finding is most consistent with a "pathways" interpretation within the life course framework, which views the early or distal childhood socio-economic environment as being important mainly through its influence on proximal socio-economic circumstances, in this case adult SEP (especially education), which in turn influences mental health status.

In other words the indirect or mediated pathway is stronger than the direct pathway, and is consistent with the substantial existing evidence that socioeconomic circumstances in adulthood are important determinants of inequalities in adult mental health.
Finally, causal mechanisms (nature and strength) are likely to vary by context, including time and place. Therefore, generalising our findings to other countries and comparing to other studies should be done cautiously.

In terms of policy, population-based research such as ours is important in providing evidence as to whether intervention programs, which aim to reduce the negative influence of serious economic adversity on adult mental health, should target their influences earlier or later in the life course. Our findings suggest that the short-term influences operating in adulthood such as unemployment and poverty are likely to be able to "circuit-break" the longer-term effects of early SEP. Nevertheless, the long-term influences, which are rooted in previous life stages, are still important as they influence mental health indirectly through current adult SEP.

\section{Acknowledgements:}

Access to the data used in this study was provided by Statistics New Zealand under conditions designed to give effect to the security and confidentiality provisions of the Statistics Act 1975 . The results presented in this study are the work of the authors, not Statistics New Zealand. The authors gratefully acknowledge the work of $\mathrm{Dr}$ Jackie Fawcett in the initial development of the SoFIE Health Study.

Funding: SoFIE Health is primarily funded by the Health Research Council of New Zealand as part of the Health Inequalities Research Programme.

\section{References}

Allan J. (2001) Review of the Measurement of Ethnicity. Classifications and Issues. Statistics New Zealand, Wellington.

Andrews G and Slade T. (2001) Interpreting scores on the Kessler Psychological Distress Scale (K10). Australian and New Zealand Journal of Public Health, 25, 494-497.

Artazcoz L, Benach J, Borrell C and Cortes I. (2004) Unemployment and Mental Health: Understanding the Interactions Among Gender, Family Roles, and Social Class. American Journal of Public Health, 94, 8288.

Barker D. (1998) Mothers, babies and health in later life. Churchill, Edinburgh.

Blakely T. (2002) Commentary: Estimating direct and indirect effects-fallible in theory, but in the real world? International Journal of Epidemiology, 31, 166-167.

Bradley RH and Corwyn RF. (2002) Socio-economic Status and Child development. Annual Review of Psychology, 53, 371-399.

Butterworth P, Rodgers B and Windsor TD. (2009) Financial hardship, socio-economic position and depression: Results from the PATH Through Life Survey. Social Science and Medicine, 69, 229-237.

Carter K, Hayward M and Richardson K. (2008) SoFIE Health Baseline Report: Study design and associations of social factors and health in Waves 1 to 3. SoFIE Health Report: University of Otago, Wellington.

Carter K, Blakely T, Collings S, Gunasekara F and Richardson K. (2009a) What is the association between wealth and mental health? Journal of Epidemiology and Community Health, 63, 221-226.

Carter K, Cronin M, Blakely T, Hayward M and Richardson K. (2009b) Cohort profile: Survey of Families, Income and Employment (SoFIE) and Health Extension (SoFIE-Health). International Journal of Epidemiology, 39,653-659. 
Cole SR and Hernan MA. (2002) Fallibility in estimating direct effects. International Journal of Epidemiology, 31, 163-165.

Davey Smith G, Hart C, Blane D and Hole D. (1998) Adverse socio-economic conditions in childhood and cause specific adult mortality: prospective observational study. British Medical Journal, 316, 1631-1635.

Davis P, Jenkin G and Coope P. (2003) New Zealand Socio-economic Index 1996: An update and revision of the New Zealand Socio-economic Index of Occupational Status. Statistics New Zealand, Wellington.

Davis P, Jenkin G, Coope P, Blakely T, Sporle A and Kiro C. (2004) The New Zealand Socio-economic Index of Occupational Status: methodological revision and imputation for missing data. Australian and New Zealand Journal of Public Health, 28, 113-119.

Davis P, McLeod K, Ransom M and Ongley P. (1997) The New Zealand Socio-economic Index of Occupational Status (NZSEI). Statistics New Zealand, Wellington.

Davis P, McLeod K, Ransom M, Ongley P, Pearce N and Howden-Chapman P. (1999) The New Zealand Socioeconomic Index: Developing and validating an occupationally-derived indicator of socio-economic status. Australian and New Zealand Journal of Public Health, 23, 27.

Dohrenwend BP, Levav I, Shrout PE, Schwartz S, Naveh G, Link BG, Skodol AE and Stueve A. (1992) Socioeconomic Status and Psychiatric Disorders: The Causation-Selection Issue. Science, 255, 946.

Fryers T, Melzer D and Jenkins R. (2003) Social inequalities and the common mental disorders: A systematic review of the evidence. Social Psychiatry and Psychiatric Epidemiology, 38, 229.

Galobardes B, Lynch JW and Smith GD. (2008) Is the association between childhood socio-economic circumstances and cause-specific mortality established? Update of a systematic review. Journal of Epidemiology and Community Health, 62, 387-390.

Galobardes B, Shaw M, Lawlor DA, Davey Smith G and Lynch J. (2006) Indicators of socio-economic position. In Oakes M, Kaufman JS. eds. Methods in Social Epidemiology. Pp 47-85. Jossey-Bass, San Franscico, CA.

Gilman SE, Kawachi I, Fitzmaurice GM and Buka SL. (2003) Socio-economic status, family disruption and residential stability in childhood: relation to onset, recurrence and remission of major depression. Psychological Medicine, 33, 1341-1355.

Gilman SE, Kawachi I, Fitzmaurice GM and Buka SL. (2002) Socioeconomic status in childhood and the lifetime risk of major depression. International Journal of Epidemiology, 31, 359-367.

Glymour M. (2007) Commentary: Selected samples and nebulous measures: some methodological difficulties in life-course epidemiology. International Journal of Epidemiology, 36, 566-568.

Glymour M and Greenland S. (2008) Causal Diagrams. In Rothaman KJ, Greenland S and Lash TL. eds. Modern Epidemiology. Third edition. Pp 103-209. Lippincott Williams and Wilkins, Philadelphia.

Graham H. (2007) Socio-economic inequalities across generations: occupation and education. Unequal Lives: Health and Socio-economic Inequalities. Open University Press, Berkshire.

Graham H and Power C. (2004) Childhood disadvantage and health inequalities: a framework for policy based on lifecourse research. Child: Care, Health and Development, 30, 671-678.

Greenland S. (2003) Quantifying Biases in Causal Models: Classical Confounding vs Collider-Stratification Bias. Epidemiology, 14, 300-306.

Greenland S. (2008) Invited Commentary: Variable Selection versus Shrinkage in the Control of Multiple Confounders. American Journal of Epidemiology, 167, 523-529.

Harper S, Lynch J, Hsu WL, Everson SA, Hillemeier M, Raghunathan TE, Salonen JT and Kaplan GA. (2002) Life course socio-economic conditions and adult psychosocial functioning. International Journal of Epidemiology, 31, 395-403.

Hausman JA. (1978) Specification Tests in Econometrics. Econometrica, 46, 1251-1271.

Hernan MA and Cole SR. (2009) Invited Commentary: Causal Diagrams and Measurement Bias. American Journal of Epidemiology, 170, 959-962.

Kessler RC, Andrews G, Colpe L, Hiripi E, Mroczek DK, Normand SLT, Walters E and Zaslavsky A. (2002) Short screening scales to monitor population prevalences and trends in non-specific psychological distress. Psychological Medicine, 32, 959-976.

Kuh D and Ben-Shlomo Y. (2004) Introduction. In Kuh D and Ben-Shlomo Y. eds. A Life Course Approach to Chronic Disease Epidemiology. Pp 3-14. Oxford University Press, Oxford.

Kuh D, Ben-Shlomo Y, Lynch J, Hallqvist J and Power C. (2003) Life course epidemiology. Journal of Epidemiology and Community Health, 57, 778-783.

Kuh D, Hardy R, Langenberg C, Richards M and Wadsworth M. (2002) Mortality in adults aged 26-54 years related to socio-economic conditions in childhood and adulthood: post war birth cohort study. British Medical Journal, 325, 1076-1080. 
Kuh D, Head J, Hardy R and Wadsworth M. (1997) The Influence of Education and Family Background on Women's Earnings in Midlife: evidence from a British national birth cohort study. British Journal of Sociology of Education 18, 385 - 405.

Kuh D, Power C, Blane D and Bartley M. (2004) Socio-economic pathways between childhood and adult health. In Kuh D and Ben-Shlomo Y. eds. A Life Course Approach to Chronic Disease Epidemiology. Pp 371-395. Oxford University Press, Oxford.

Kuh D and Wadsworth M. (1991) Childhood Influences on Adult Male Earnings in a Longitudinal Study. The British Journal of Sociology, 42, 537-555.

Laaksonen M, Rahkonen O, Martikainen P and Lahelma E. (2005) Socio-economic Position and Self-Rated Health: The Contribution of Childhood Socio-economic Circumstances, Adult Socio-economic Status, and Material Resources. American Journal of Public Health, 95, 1403-1409.

Lorant V, Croux C, Weich S, Deliege D, Mackenbach J and Ansseau M. (2007) Depression and socio-economic risk factors: 7-year longitudinal population study. British Journal of Psychiatry, 190, 293-298.

Lorant V, Deliege D, Eaton W, Robert A, Philippot P and Ansseau M. (2003) Socio-economic Inequalities in Depression: A Meta-Analysis. American Journal of Epidemiology, 157, 98-112.

Luo Y and Waite LJ. (2005) The Impact of Childhood and Adult SES on Physical, Mental, and Cognitive Well-Being in Later Life. The Journals of Gerontology, 60B, S93.

Lynch JW, Kaplan GA and Salonen JT. (1997) Why do poor people behave poorly? Variation in adult health behaviours and psychosocial characteristics by stages of the socio-economic lifecourse. Social Science and Medicine, 44, 809-819.

Mckenzie S and Carter K. (2009) Are retrospective measures of childhood socio-economic position in prospective adult health surveys useful? Australasian Epidemiologist, 16, 22-24.

Melchior M, Moffitt TE, Milne BJ, Poulton R and Caspi A. (2007) Why Do Children from Socio-economically Disadvantaged Families Suffer from Poor Health When They Reach Adulthood? A Life-Course Study. American Journal of Epidemiology, 166, 966-974.

Miech RA, Caspi A, Moffitt TE, Wright BR and Silva PA. (1999) Low Socio-economic Status and Mental Disorders: A Longitudinal Study of Selection and Causation During Young Adulthood. The American Journal of Sociology, 104, 1096-1131.Ministry of Health (2008) A Portrait of Health. Key Results of the 2006/07 New Zealand Health Survey. Minstry of Health, Wellington.

Muntaner C, Eaton W, Meich R and O'Campo P. (2004) Socio-economic position and major mental disorders. Epidemiologic Reviews, 25, 53-62.

Oakley Browne MA, Wells JE and Scott KM. (2006) Te Rau Hinengaro: The New Zealand Mental Health Survey. Ministry of Health, Wellington.

Oakley Browne MA, Wells JE, Scott KM and McGee MA. (2010) The Kessler Psychological Distress Scale in Te Rau Hinengaro: the New Zealand Mental Health Survey. Australian and New Zealand Journal of Psychiatry, 44, 314-322.

Phongsavan P, Chey T, Bauman A, Brooks R and Silove D. (2006) Social capital, socio-economic status and psychological distress among Australian adults. Social Science and Medicine, 63, 2546-2561.

Piccinelli M and Wilkinson G. (2000) Gender differences in depression: Critical review. The British Journal of Psychiatry, 177, 486-492.

Poulton R, Caspi A, Milne BJ, Thomson WM, Taylor A, Sears MR and Moffitt TE. (2002) Association between children's experience of socio-economic disadvantage and adult health: a life-course study. The Lancet, 360, 1640-1645.

Power C, Atherton K, Strachan DP, Shepherd P, Fuller E, Davis A, Gibb I, Kumari M, Lowe G, Macfarlane GJ, Rahi J, Rodgers B and Stansfeld S. (2007) Life-course influences on health in British adults: effects of socioeconomic position in childhood and adulthood. International Journal of Epidemiology, 36, 532-539.

Power C and Manor O. (1992) Explaining social class differences in psychological health among young adults: a longitudinal perspective. Social Psychiatry and Psychiatric Epidemiology, 27, 284-291.

Power C and Matthews S. (1997) Origins of health inequalities in a national population sample. The Lancet, 350, 1584-1589.

Power C, Stansfeld SA, Matthews S, Manor O and Hope S. (2002) Childhood and adulthood risk factors for socioeconomic differentials in psychological distress: evidence from the 1958 British birth cohort. Social Science and Medicine, 55, 1989-2004.

Repetti RL, Taylor SE and Seeman TE. (2002) Risky Families: Family Social Environments and the Mental and Physical Health of Offspring. Psychological Bulletin, 128, 330-366.

Rothman KJ, Greenland S and Lash TL (2008) Modern Epidemiology. Lippincott Williams \& Wilkins, Philadelphia. Salmond C and Crampton P. (2002) NZDep2001 Index of Deprivation. University of Otago, Wellington. 
Singh-Manoux A, Ferrie JE, Chandola T and Marmot M. (2004) Socio-economic trajectories across the life course and health outcomes in midlife: evidence for the accumulation hypothesis? International Journal of Epidemiology, 33, 1072-1079.

Skapinakis P, Weich S, Lewis G, Singleton N and Araya R. (2006) Socio-economic position and common mental disorders: Longitudinal study in the general population in the UK. British Journal of Psychiatry, 189, 109-117.

Stansfeld SA, Head J, Fuhrer R, Wardle J and Cattell V. (2003) Social inequalities in depressive symptoms and physical functioning in the Whitehall II study: exploring a common cause explanation. Journal of Epidemiology and Community Health, 57, 361-367.

Stansfeld SA, Head J and Marmot MG. (1998) Explaining social class differences in depression and well-being. Social Psychiatry and Psychiatric Epidemiology, 33, 1.

Statistics New Zealand (2001) New Zealand Standard Classification of Occupation 1999. Statistics New Zealand, Wellington.

Statistics New Zealand (2008) Survey of Family, Income and Employment: Wave Four September 2006. Wellington: Statistics New Zealand.

Weich S and Lewis G. (1998a) Material standard of living, social class, and the prevalence of the common mental disorders in Great Britain. Journal of Epidemiology and Community Health, 52, 8-14.

Weich S and Lewis G. (1998b) Poverty, unemployment, and common mental disorders: population based cohort study. British Medical Journal 317, 115-119. 
